\title{
Research on Vehicle Detection and Tracking Algorithm Based on the Methods of Frame Difference and Adaptive Background Subtraction Difference
}

\author{
Yiqin Cao*, Xiao Yun, Tao Zhong and Xiaosheng Huang \\ School of Software, East China Jiaotong University, Nanchang, 330013, China \\ ${ }^{*}$ Corresponding author
}

\begin{abstract}
This paper proposed methods of vehicle detection and tracking algorithm in real-time traffic. In the detection of realtime moving vehicle, vehicle areas would be determined through road line detection. Then, the main color information of moving and non-moving area would be obtained through frame difference. Filling the main color information in vehicle moving area would lead to a similar background image. At last, moving vehicles would be determined through adaptive Background Subtraction difference. In the tracking of moving vehicles, firstly, all characteristic corners can be got by using Harris detection. Then, all characteristic corner set in the separate moving area would be collected through cluster analysis. For each characteristic individual corner set can generate a circle embracing all characteristics, some problems like vehicle barrier could be analyzed by using the radius of characteristic circle. At last, conduct feature matching tracking by using the center of feature circle. Experimental result shows that the improving algorithm can extract all moving objects, which was endowed with strong background adaptability and better real-time performance.
\end{abstract}

Keywords-target detection; frame difference method; background subtraction difference method; harris corner detection; clustering analysis

\section{INTRODUCTION}

Video-based detection and tracking of moving vehicles has been a hot spot in the study of computer vision. Detection and tracking of moving vehicles is an important part of the intelligent transportation system. The key techniques of that includes vehicle detection, image pre-process, vehicle tracking, identification and etc [1-2]. At present, the major approaches to detect moving vehicles are inter-frame difference method, Background Subtraction difference method, optical flow method and etc [3-4].

Background Subtraction difference method [5-6] applies the subtraction of current image sequence and the reference background model to detect the target of moving vehicle, which is fast and simple, but moving target can only be detected by using the clear background image. When there is some fluctuations of test conditions,such as illumination change, the accuracy of detection will be influenced. Interframe difference method [7-8] applies the difference of two or three adjacent images to obtain the moving target area, which is of strong adaptability and robustness to the moving target. But when dealing with slow moving or stationary target, residual detection may appear. The optical flow method [9-10] is to detect the characteristics of every pixel in each frame of image sequence. It has disadvantages of great amount of calculation, poor real-time performance and poor anti-noise performance, which imposes a negative influence on the detection. Based on the integration of inter-frame difference and Background Subtraction difference methods, the approach to deal with background frame has been updated, but it doesn't update for the complete background image, which means the traditional method doesn't get the actual background image. It has a negative effect on vehicle tracking. When a vehicle is in the normal process of moving, it can be detected and tracked ideally through traditional methods. But when there is a sudden stop of the vehicle in the process of moving, traditional methods will treat the vehicle as background information and lost the target. When the stationary vehicle move again, it will be regarded as a new target, thus this tracking method can not meet to the requirements of tracking. Reference Wang etc.[10] proposed a new algorithm to detect and track moving vehicle which is a combination of frame difference and optical flow. This algorithm applies the inter-frame difference method to detect the moving object movement area, calculate the nonzero light flow in difference map, and applies the optical flow field to track moving targets, but this method needs a large amount of calculation and has a complex calculation process. Based on the Background Subtraction difference and the frame difference methods, Reference Li G etc.[11] raised a new method to detect the moving target. Aimed at tracking moving target under the static background, It combines the accuracy in monitoring object of Background Subtraction difference method of with strong adaptability to the light of frame difference method, which improves the stability, but this method is not very ideal which may cause errors sometimes when dealing with complex motion detection.

This paper proposes a method which obtains the driving area of vehicle through road line detection, captures the main color information of motion area and non-motion area of moving vehicle through inter-frame difference method, fills the motion area with major color information of non-motion area to obtain an approximate background image and captures the moving vehicle through Background Subtraction difference. Then all feature corner points of the moving vehicle can be obtained by Harris detection. The feature corner sets of all the separated motion areas can be obtained by cluster analysis and each feature corner set generates a feature circle containing all feature points which can be applied to analyze the vehicle 
occlusion. The circle of the feature center can be used to track feature. This method solves the problem of dynamic tracking and occlusion issues of vehicles in complicated situations, and improves the real - time performance and accuracy of vehicle detection and tracking.

\section{Moving Vehicle Detection Method BASEd on TECHNIQUES OF INTER-FRAME DIFFERENCE AND BACKGROUND SUBTRACTION DIFFERENCE}

Determine the target vehicles on the areas different from road through analysis on all the color information of the road according to actual road condition. In this paper, the method of frame difference, Background Subtraction difference and color information contrast were applied to detect the moving target vehicles. First of all, we should detect road line on the first frame of moving video and determine the vehicle running area in accordance with the road line. Then, we can get the moving area and non-moving area of the picture using frame difference method and view the non-moving area as background area. For the road information of moving area shares similar features, we can quickly obtain an approximate background image by filling the basic color information of the non-moving region into the moving region in the image. Finally, the Background Subtraction difference is used to determine the moving vehicle.

\section{A. Determination of Vehicle Running Area}

According to actual road condition, vehicle running area has its own particularity that the main color information between the road lines are basically the same with little nuance. Verifying vehicle running areas can reduce the impact of surrounding environment and greatly improve the accuracy of detection. In this method the main color information of vehicle running areas can be used to detect the moving vehicles.

Because light has a certain influence on road information, and many trees and obstacles around roads would the light, it is to some extent difficult to get road information from lines. However, the greatest difference between road markings and other obstacles is that the former is a straight line. Therefore, in this paper, road line detection was carried out through Hough transformation[12-13]. The main vehicle running areas can be determined by connecting the lines. Firstly, the image should be grayed out and the noise in the video information should be removed by median filtering. Then, the edge is obtained by edge detecting Prewitt operator. Finally, the line in the edge is obtained by Hough algorithm. During filming the road, the road line is generally shown in a slash, which can remove the vertical and horizontal road lines to improve the accuracy of road detection. The marking lines on the same road may be divided into a plurality of shorter road marking lines at the time of detection due to occlusion, breakage or detection sensitivity and other reasons. The divided road marking lines can be restored to the original whole one by fitting method to improve the reliability of detection. Finally, the longest road marking lines on both sides should be linked orderly so as to determine the vehicle running area.

\section{B. Quickly Obtaining Background through Frame Subtraction}

The traditional method to obtain the background is multiframe mean. In practice, the method can provide a more realistic background model, but its real-time performance is poor. This paper presents a method of frame difference to quickly get the background model, which is simple and has good real-time performance.

Firstly, determining the vehicle running area by using the method of Section 1.1.on the assumption that the image of the two consecutive frames are $I_{t}(x, y)$ and $I_{t-1}(x, y)$ respectively. Then, processing the image of two successive frames at the same time to obtain the actual trajectory of the vehicle and getting the two resulted frames image of $\bar{I}_{t}(x, y)$ and $I_{t-1}(x, y)$.

Next, making a difference between the two images so as to determine the running areas of the moving vehicle:

$$
\operatorname{CarR}_{t}(x, y)=\bar{I}_{t}(x, y)-\bar{I}_{t-1}(x, y)
$$

Thresholding the vehicle region and obtain a binary image:

$$
\operatorname{BiCarR}_{t}(x, y)= \begin{cases}1 & \text { if }\left|\operatorname{CarR}_{t}(x, y)\right|>\operatorname{CarTh} \\ 0 & \text { else }\end{cases}
$$

Formula CarTh means threshold value. The binary image got through threshold value processing has defects of region segmentation. The moving vehicle region $\operatorname{BiCarR}_{t}^{\text {new }}(x, y)$ with better connectivity is obtained by closed operation in the paper. Then, process $\bar{I}_{t}(x, y)$ according to the vehicle region to determine whether it is a moving one. If it is, it will be set to 0 . Otherwise, it should be kept its original information unchanged so as to obtain non-moving region of road area:

$$
\operatorname{RrodR}_{t}(x, y)=\left\{\begin{array}{lc}
\bar{I}_{t}(x, y) & \text { if } \operatorname{BiCarR}_{t}^{\text {new }}(x, y)=0 \\
0 & \text { else }
\end{array}\right.
$$

The gray information of the main road area $\operatorname{RrodR}_{t}(x, y)$ in the non-moving region is analyzed and the gray information of the area is obtained by the color histogram according to the color information of road ground. Then, the important gray information MainGray ${ }_{t}$ can be obtained through weighted mean.

Finally, the main gray information is filled into the moving area to complete the background acquisition:

$$
B g_{t}=\left\{\begin{array}{lc}
\text { MainGray }_{t} & \text { if } \operatorname{BiCarR}_{t}^{\text {new }}(x, y)=1 \\
\bar{I}_{t}(x, y) & \text { else }
\end{array}\right.
$$

Compared with the traditional multi-frame difference method, the frames difference method applied in this paper does not need to acquire prior knowledge and has stronger real- 
time performance. Compared with the Gaussian model, it is more simple and efficient.

\section{Self-Adaptive Background Subtraction Difference}

Background Subtraction difference method[3-4] requires first setting up the Gaussian background model as the background image, then calculating the difference of pixel brightness between current video sequence image and the known background image and taking the absolute value. At last, the pixels of current frame that differ from background image will be obtained. The obtained video frame image is divided into a moving area and background area. In the obtained difference image, the low gray-value area corresponds to the background of the current frame image and the high gray-value area corresponds to the moving part. The background area and the motion area will be distinguished by setting the threshold value. The threshold value also determines the sensitivity of the target detection. Selecting an appropriate threshold value has a greater impact on the target detection.

Background Subtraction difference can better obtain the information of moving vehicles, but does not have the robustness of illumination. The use of adaptive Background Subtraction difference can better solve this problem. In this paper, the background is updated adaptively based on the parameter information obtained from the frame difference. So that the background has better illumination robustness and can detect the target vehicle better.

Based on influences of light on the ground information, it has a great influence on road area in formula (3), and finally on the Main Gray information. Therefore, it only needs to observe the changes of MainGray to judge whether the background needs to be renewed. If the change of the gray scale is greater than a certain threshold value, the background will be updated in accordance with formula (4), which can solve the problem of illumination.

Assuming that the road region of the image of the $t+1$ frame has been obtained in accordance with Formula (3), the MainGray information of the region is MainGray ${ }_{t+1}$, which is obtained through the color histogram. So, the background adaptive updating method is:

$$
B g_{t+1}= \begin{cases}B g_{\text {main }} & \mid \text { MainGray }_{t+1}-\text { MainGray }_{\text {main }} \mid<M G T h \\ B g_{t+1}^{\text {new }} & \text { otherwise }\end{cases}
$$

Here, $B g_{\text {main }}$ and MainGray ${ }_{\text {main }}$ are the background of the first few frames for the Background Subtraction difference and the main gray-scale information. MGTh is the threshold value of gray-scale change. As long as the gray-scale change does not exceed the threshold value range, the background does not need to be updated. Otherwise, it should be updated. Then, regain the new background of $B g_{t+1}^{\text {new }}$ through the methods of 1.2 (5) and arrive at

$$
B g_{\text {main }}=B g_{t+1}^{\text {new }} \text { and MainGray } \text { main }=\text { MainGray }_{t+1} .
$$

Next, the background adaptive updating of the next frame is performed by the formula (5).

The obtained background is assumed to be $B g_{t}(x, y)$. The Background Subtraction difference image of $B F_{t}(x, y)$ can be obtained through the current frame being subtracted from the background image.

$$
B F_{t}(x, y)=\left\{\begin{array}{lc}
\bar{I}_{t}(x, y) & \left|\bar{I}_{t}(x, y)-B g_{t}(x, y)\right|>B F T h \\
0 & \text { otherwise }
\end{array}\right.
$$

Here, BFTh is threshold value of the moving area. $B F_{t}(x, y)$ is the moving area in the foreground.

\section{A FAST MATCHING METHOD}

Point-to-point matching tracking and the occlusion of image extraction in process of moving need to be overcome in feature-based tracking algorithm. This paper raises a fast matching method to solve those two issues. This method is aimed to solve two issues. The first one is real-time issue. On the basis of the particularity of vehicle appearance, a vehicle is approximately rectangular or rhombus in video sequence. After Harris corner detection[14], the center of gravity of the vehicle can be obtained through analyzing detected corner set. In the video sequence, because the position of the vehicle in each frame doesn't change too much, the tracking can be achieved according to the nearest gravity point of vehicle in the next frame of the image. Thus corner tracking can be converted to the gravity point tracking. The second issue is block issue. This paper proposes that minimum circle needs to be introduced, whose center is the gravity center and which contains the feature corner set and is called the feature circle. When a target vehicle is in the process of moving, the distance between the target vehicle and camera will be changed and the shape of target vehicle will change too, so its feature circle radius will be updated adaptively. When the target vehicle stops, the radius will stay the same. When the target vehicle is far away from the camera, the radius will become smaller and Vice versa.Through calculating, the radius of the next feature circle can be predicted and called the forecast radius. Through the comparison between the forecast radius and the true radius, it can be easier to recognize whether the vehicle is blocked. When the vehicle occlusion happens, the original two motion areas will merged into one and the real radius will suddenly become larger. At this time, center of gravity can be predicted through particle filtering[15]and maintain adaptive forecast radius of the vehicle to track the vehicle.

\section{A. Matching Method Based on the Center of Gravity}

On account of the corners containing a large amount of vehicle information, and information of corner points does not change much in two consecutive frames when corner detection is accurate,so the vehicle tracking can be achieved by corner matching. Corner matching methods can be divided into two branches [16]. One is the matching of the corresponding points, by which the corner set of two consecutive images is obtained, and then the similarity of the corner points is applied to 
determine the correspondence between each corner point of the two point sets. When a certain number of matching is satisfied, the matching relationship of the two point sets can be determined. The other is the corner set matching, which doesn't need the establishment of the corresponding corners, and only need to calculate the distance between the corners set, which means to do the match through the degree of similarities of corner sets. Because the corner set matching method is relatively sensitive to the rotation and scale transformation of the object, this paper applies the corresponding point matching algorithm. Although the corresponding point matching algorithm is of a relatively high matching accuracy for objects with simple shapes, the shape of the vehicle is more complex than the ordinary objects, and the motion of the vehicle is unpredictable. When a vehicle makes turns or gets blocked, the original corner set may be changed after corner detection, and the matching of corner points will be greatly disturbed. And the corresponding point matching algorithm needs to match every corner point in the corner set, which is inefficient and can not meet the real-time requirement of tracking. Inspired by the tracking of the corresponding points in the region tracking, a gravity center matching algorithm is proposed according to the vehicle shape information.

First, the gravity center of motion area can be obtained through feature corner point, and can be pre-matched with the gravity center of a frame in the image. The feature point set of the No. $t$ frame image can be obtained by the Harris corner detection, the feature point set of i separate motion regions: $M_{t}^{i}(x, y)$ can be obtained by clustering, and the points of each motion region: $C_{t}^{i}(x, y)$ can be obtained by analyzing those feature point sets. According to the area of the moving vehicle is gravity center with the shape of rectangular or diamond, four points can be used to determine the center of gravity:

$$
\begin{aligned}
& C_{t}^{i}(x)=\frac{\max \left(M_{t}^{i}(x)\right)+\min \left(M_{t}^{i}(x)\right)}{2} \\
& C_{t}^{i}(y)=\frac{\max \left(M_{t}^{i}(y)\right)+\min \left(M_{t}^{i}(y)\right)}{2}
\end{aligned}
$$

Through the above two equations, the gravity point $C_{t}^{i}(x, y)$ of the No. i moving area of the No. $t$ frame image can be obtained. Through the same method, the gravity enter $C_{t+1}^{i}(x, y)$ of each moving area of the next frame can be obtained. The position of the moving vehicle in two consecutive frames will not change too much. Thus the two nearest consecutive images can be matched to select the shortest point to make the prematching. Then the occlusion will be determined. By this method, the matching of each feature corner, the complexity of the operation and the computing time can be reduced and the tracking effect can be improved.

\section{B. Vehicle Occlusion Judgments}

When the occlusion of the vehicle happens, the radius of the feature circle generated by the feature corner is used to judge the occlusion, and the final accurate matching will be made. By using the feature point set $M_{t}^{i}(x, y)$ to obtain a minimum circle with the center of the circle $C_{t}^{i}(x, y)$, which contains all the feature points in the motion region and is called the feature circle. The radius of the circle is $R_{t}^{i}$. In the video sequence, when the target vehicle is far away from the camera, the radius will become smaller,and vice versa. The radius of the feature circle $R_{t}^{i}$ can be updated as:

$$
\bar{R}_{t}^{i}=\alpha^{ \pm 1} \times R_{t-1}^{i}
$$

where $\alpha$ can be obtained through the experiment, and the value is usually 1.15 . When the vehicle is close to the camera, the value is $\alpha^{+1}$, and the vehicle is far away from the camera, the value is $\alpha^{-1}$.

$\bar{R}_{t}^{i}$ is the radius of the feature circle through estimation, the radius of the actual feature circle through analyzing is $R_{t}^{i}$, and it is relatively simple to determine the occlusion and separation by comparing the two radii. When the vehicle occlusion occurs, the original two motion areas will be merged into one, that is, the radius of the feature circle will suddenly become very large. When the vehicle separation occurs, the original block of the moving area will be separated into two parts, that is, the radius of the feature circle will suddenly become smaller. Specific judgments are as follows:

1) Normal driving: $\left|\frac{R_{t}^{i}-\bar{R}_{t}^{i}}{R_{t}^{i}}\right|<r T h$;

2) Vehicle occlusion: $\left|\frac{R_{t}^{i}-\bar{R}_{t}^{i}}{R_{t}^{i}}\right|>r T h$ and $R_{t}^{i}>\bar{R}_{t}^{i}$;

3) Vehicle separation: $\left|\frac{R_{t}^{i}-\bar{R}_{t}^{i}}{R_{t}^{i}}\right|>r T h$ and $R_{t}^{i}<\bar{R}_{t}^{i}$

Wherein, $r T h$ is the a radius decision threshold. When the vehicle is the normal moving, the gravity center matching method can be directly used. Tracking can be achieved by matching the nearest points of $C_{t}^{i}(x, y)$ and $C_{t+1}^{i}(x, y)$. When the vehicle is blocked, $C_{t}^{i}(x, y)$ will be processed through the particle filtering and the position of the next frame $\bar{C}_{t+1}^{i}(x, y)$ will be substituted by $C_{t+1}^{i}(x, y)$ and the predicted radius is used to substitute the actually obtained radius, i.e., $R_{t+1}^{i}=\bar{R}_{t+1}^{i}$. When the vehicle is separated, the predicted gravity center when occlusion occurs is taken as the gravity center, and the next frame is tracked through the optimized matching method.

\section{EXPERIMENTAL RESULTS AND DISCUSSION}

Simulation results from MATLAB R2012b software programming, computer hardware configuration is Intel Core i5/3.50 GHz/4.00GB of memory. In the process of simulation, inter-frame difference threshold is 0.156 ,gray threshold value 
of 0.03,background Subtraction difference threshold is 0.15 .The video resolution is $320 \times 224$ according to a highway vehicle motion video which used as an experiment object.

This paper proposes a method which based on the fusion of inter-frame difference and background subtraction difference. First of all, the proposed method uses the Rough road line detection for the first image. Secondly, it determines the main driving area of the vehicle through the road line, then it obtains two long road lines about two sides through the experiment, next connect the two road routes in an orderly manner, finally get the determined vehicle driving area.

This paper proposes a method which based on inter-frame difference and background subtraction difference fusion. First of all, use the Rough road line detection for the first image, secondly, determine the main driving area of the vehicle through the road line. Then obtained two long road lines about two sides through the experiment, next connect the two road routes in an orderly manner, finally get the determined vehicle driving area. As shown in Figure I.

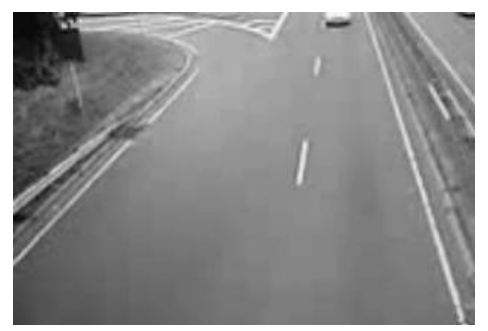

(a)The first frame image

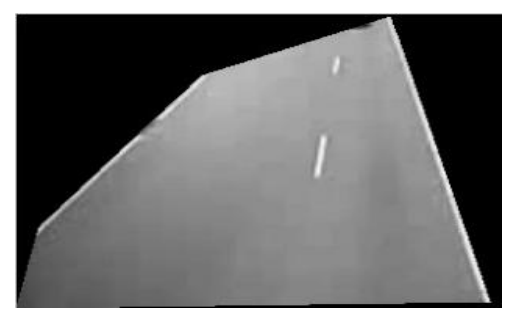

(b)Vehicle driving area

\section{FIGURE I. DETERMINE VEHICLE DRIVING AREA THROUGH THE ROUGH ROAD LINE DETECTION}

It is found that the vehicle driving area can remove the influence of buildings and trees beside the road on the target detection, and it is the base of the background model through the inter-frame difference.

After vehicle running area is determined, it is assumed that the moving area and the non-moving area of the image would be obtained through conducting frame difference on the 13th frame and the 14th frame image, as shown in Figure II (a). The black part of vehicle running area is moving area, and the rest is the non-moving area. Then, the color histogram is used to analyze the color information of the non-moving area, as shown in Figure II (b). The main gray information of nonmoving area can be obtained by taking a weighted average of the gray value greater than 1000 . And then, we can obtain the background image by filling the gray information into the moving area of the image, as shown in Figure II (c).

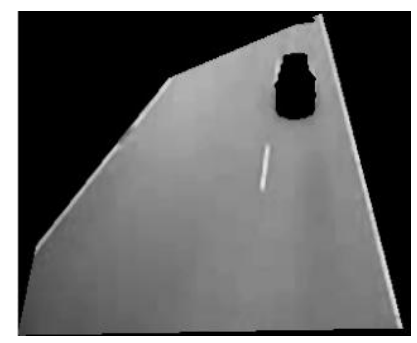

(a) non-moving area obtained through frame difference

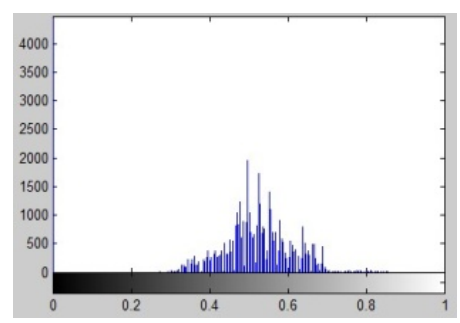

(b) gray histogram of non-moving area

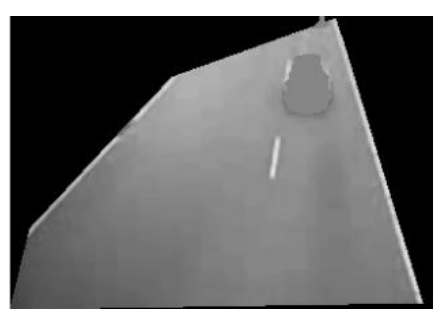

(c) Background image

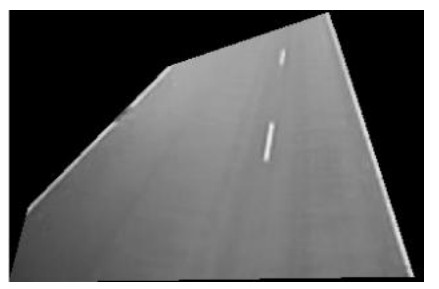

(d) Background obtained through average frame

\section{FIGURE II. THE BACKGROUND IMAGE OBTAINED THROUGH INTER- FRAME DIFFERENCE}

Figure II. c and Figure II. d are the background obtained through the proposed method and traditional multi-frame averaging method respectively. From the visual aspect, although the background image obtained through frame difference is less specific and smooth than that through average, this algorithm is simpler and has better real-time performance.

After the background image is obtained through the interframe difference, the moving object can be detected by using the background subtraction difference, as shown in Figure III(b).Compare the traditional background subtraction difference with the goal obtained by the method proposed in this paper has more details about the vehicle information, which has a good significance for describing the target as well as rebuilding the vehicle model. It also provides a good basic for the follow-up vehicle tracking. 


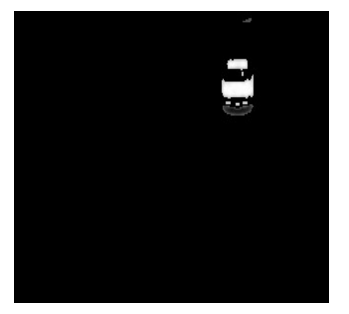

(a) get the target by traditional Background Subtraction difference approach

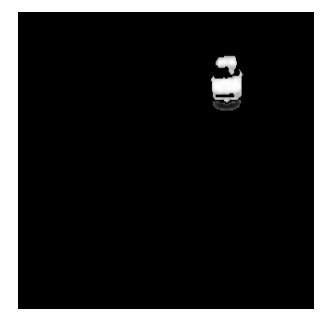

(b) get the target by the proposed approach

\section{FIGURE III. CONTRAST FIGURE OF TARGET DETECTION}

According to the experiment results as follow,it shows that the results using the algorithm proposed in this paper has made a great improvement in accuracy and timeliness. Compare time efficiency and accuracy in this paper by calculating discrimination(RR),error detection rate(EDR) and background construction time(f/ms) as follow.

$$
R R=\frac{T A}{T A+F N}, E D R=\frac{F A}{T A+F A}
$$

TA means the number of actual detected moving target, FA means the number of error detected static target, FN means the number of undetected number, $T(\mathrm{f} / \mathrm{ms})$ means background construction time, $\mathrm{f}$ means frame, as shown in Figure IV.

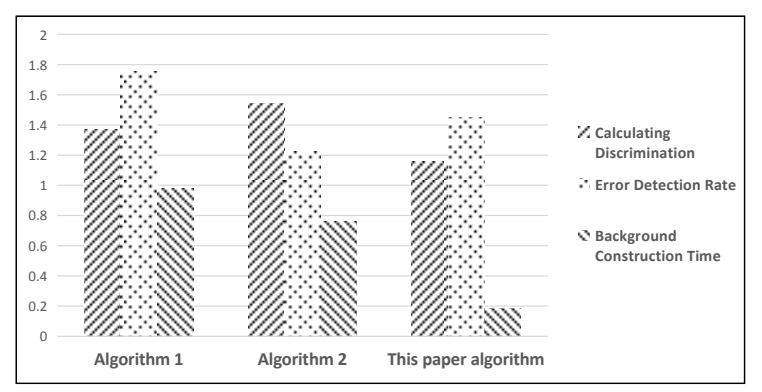

FIGURE IV. COMPARE RR,FDR AND T

The algorithm from one to three respectively indicates the Literature [10], Literature [11] and the algorithm proposed in this paper respectively.All these three algorithms can detect the moving object effectively from the figure, but recognition rate of algorithm two is relatively lower;algorithm two results a high rate of false detection when the background changes, but the proposed adaptive background updating algorithm in this paper overcomes the influence of illumination changes with the lowest error rate. In aspect of processing time $\mathrm{T}(\mathrm{f} / \mathrm{ms})$ of each frame, the proposed algorithm is significantly better than the other two algorithms, gaining lower computational complexity, shorter computing time and higher efficiency.

The advantages of the approach in this paper can be concluded from the experimental results above: (1) Simple, and good real-time performance; (2) Strong anti-interference ability, and higher detection accuracy by determining the vehicle area using the road line and excluding the impact of the surrounding environment; (3) compared to the traditional vehicle detection algorithm based on inter-frame difference and background subtraction ,this approach can obtain the determined background model :when vehicles suddenly stop, it will not immediately start background information computing, but when vehicles have stopped for a long time, it just start background information processing. The proposed algorithm is not only conducive to the continuity of target tracking, but also accord with the actual situation.(4) In this paper, acquired background through the proposed algorithm can be self-adaptive, which can overcome the influence of illumination change.

Assume that it can get the feature point set of frame $t$ picture through Harris detection of feature corner set, as shown in Figure V and Figure VI.

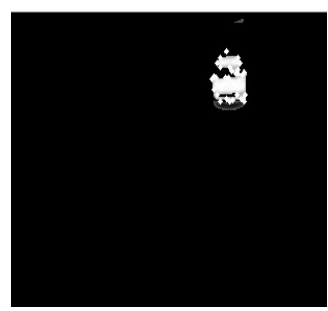

FIGURE V. HARRIS CORNER DETECTION

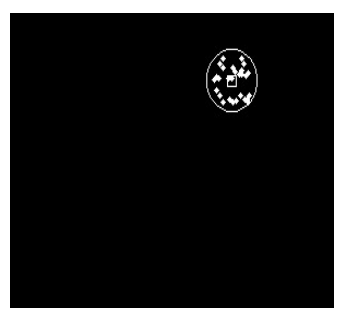

FIGURE VI. FEATURE CIRCLE OF FRAME T

Use gravity Center Matching Method when driving normally.Match the nearest point of $C_{t}^{i}(x, y)$ and $C_{t+1}^{i}(x, y)$ to achieve the target tracking, as shown in Figure VII and Figure VIII.

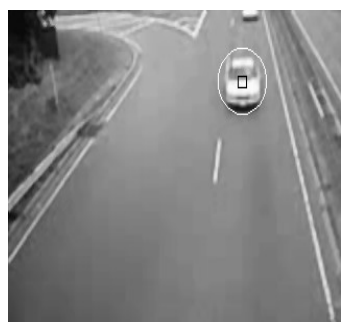

FIGURE VII. VEHICLE LOCATION OF FRAME T 


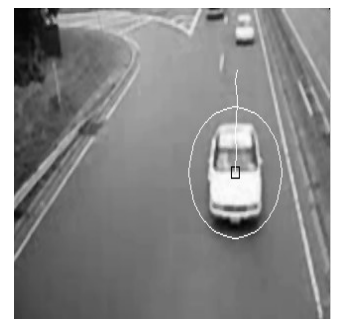

FIGURE VIII. VEHICLE TRACKING LINE OF FRAME T+1

To sum up, when dealing with vehicle detection, the proposed approach not only holds the strength of traditional methods with simple and effectiveness, but also gets the approximate background image quickly, as the Background Subtraction difference provides a basis to improve the accuracy of tracking. When dealing with vehicle tracking, our approach greatly reduces the computational complexity of the matching, and transforms the traditional corner matching into gravity circle matching with greatly improving the real-time tracking performance. The proposed approach of feature circle occlusion judgment significantly improves the accuracy of tracking.

\section{CONCLUSION}

The inter-frame difference method and the Background Subtraction difference method are the main methods used in the current vehicle detection and tracking. In order to overcome their shortcomings and take their advantages, this paper presents an algorithm of vehicle detection and tracking based on inter-frame difference method and Background Subtraction difference method which obtains the motion area and the non-motion area in the image through inter-frame difference method, takes the non-motion area approximated as background, fills the motion area in the image with major color information of non-motion area to gain an approximate background image because the ground information of moving area is almost consistent, and captures the moving vehicle through Background Subtraction difference method. Based on the detection of the vehicle, the feature points and the position parameters of the target vehicle can be extracted. The feature points of next frame can be obtained by the same method.Then the correspondence between the feature points of the previous frame and those of the current frame is established. This paper proposes a new method to tackle vehicle tracking occlusion on the basis of optimized matching algorithm, which reduces the computational complexity and improves the real-time tracking performance through using gravity center to track which is generated by the corner point set. Simultaneously, a minimum feature circle containing all corner points is generated by the corner set, and the occlusion can be analyzed by the radius of feature circle, thus tracking accuracy is improved and matching tracking can be realized. The feasibility of the method proposed by this paper is verified by the simulation results.

\section{ACKNOWLEDGMENT}

The work described in this paper was partially supported by the National Nature and Science Foundation of China under Grants 61365008.the Grants GJJ150522 and GJJ150526 from the Bureau of Education of Jiangxi and Humanity and Social Science Foundation of Ministry of Education of China under Grants 15YJA860013, This project was supported by the Innovation Fund Designated for Graduate Students of Jiangxi Province YC2015-S259.

\section{REFERENCES}

[1] Li X, Yao X C, Murphey Y L, et al. A Real-Time Vehicle Detection and Tracking System in Outdoor Traffic Scenes.[C]// Pattern Recognition, 2004. ICPR 2004. Proceedings of the 17th International Conference on. IEEE, 2004:761-764.

[2] Fossati A, Schönmann P, Fua P. Real-time vehicle tracking for driving assistance[J]. Machine Vision \& Applications, 2011, 22(2):439-448.

[3] Naimish Kasundra; Krishna K. Warhade. Performance evaluation of object detection and tracking method under illumination variation.2014 Annual IEEE India Conference (INDICON).16,DOI:10.1109/INDICON.2014.7030369

[4] HANG ShenZhi.Research Of Moving Target Detection And Tracking Technique Under Shifting Background[D]. Zhejiang University,2011

[5] Lu Z, Kong D, Li X, et al. A Method for Moving Object Detection Based on Background Subtraction and Three- Frame Differencing[J]. Computer Measurement \& Control, 2013.

[6] BQ Li;GHRen,et al.Moving target detection and tracking interactive algorithm based on acoustic image[J]IEEE/OES China Ocean. Acoustics (COA).1 - 5, DOI: 10.1109/COA.2016.7535725

[7] Prem Kumar Bhaskar;Suet-Peng Yong;et.al.Enhanced and effective parallel optical flow method for vehicle detection and tracking.2015 International Symposium on Mathematical Sciences and Computing Research (iSMSC).138 - 143, DOI: 10.1109/ISMSC.2015.7594042

[8] Xiong C, Lin L. Research on high-definition video vehicles location and tracking[C]// International Symposium on Distributed Computing \& Applications To Business. IEEE Computer Society, 2010:487-490.

[9] Jing X U, Zhang H, Zhang X J. IR Motive Detection Using Image Subtraction and Optical Flow[J]. Computer Simulation, 2012, 29(6):248252.

[10] Wang, Z. (2012). A new algorithm of moving vehicle detection and tracking based on combining frame difference method with optical flow technique. Computer Applications \& Software, 29(5), 117-113.

[11] Li G. New moving target detection method based on background differencing and coterminous frames differencing[J]. Chinese Journal of Scientific Instrument, 2006, 27(8):961-964.

[12] Hao X, Yang M, Yang X. Vehicle detection based on self-adaptive background updating[C]// International Symposium on Instrumentation \& Measurement, Sensor Network and Automation. IEEE, 2012.

[13] Feng Z, Chao Y, Wang C, et al. Circle detection and its number identification in complex condition based on random Hough transform[J]. Yi Qi Yi Biao Xue Bao/chinese Journal of Scientific Instrument, 2013, 34(3):622-628.

[14] Zhang C P, Wei X G, elecEngineering. Rectangle detection based on Harris corner[J]. Optics \& Precision Engineering, 2014, 152(3):34793491.

[15] TC Li, HQ Fan, SD Sun.Particle filtering: Theory, approach, and application for multitarget tracking[J].Acta Automatica Sinica, 2015(12):1981-2002.

[16] Minglei L, Liu D, Zeng Z. Novel image retrieval method based on improved K -means algorithm[J]. Journal of Computer Applications, 2013. 